
\title{
25 Research Square \\ Intimately Coupling Photocatalytic Optical Fibers and Biofilm for Rapid and Sustainable Degradation of 4-Chlorophenol
}

\section{Jilin Yuan}

Chongqing University of Technology

\section{Linyang Li}

Chongqing University of Technology

\section{Chuanbao Xiao}

Chongqing University of Technology

Nianbing Zhong ( $\square$ zhongnianbing@163.com )

Chongqing University of Technology https://orcid.org/0000-0001-9442-3183

\section{Dengjie Zhong}

Chongqing University of Technology

\section{Haixing Chang}

Chongqing University of Technology

\section{Yuanyuan He}

Chongqing University of Technology

\section{Tiancong Li}

Chongqing University of Technology

\section{Research Article}

Keywords: N-doped TiO2, Photocatalytic optical fiber, Microalgal biofilm, Photocatalysis, Biodegradation, 4-chlorophenol

Posted Date: April 6th, 2021

DOI: https://doi.org/10.21203/rs.3.rs-339097/v1

License: (c) (i) This work is licensed under a Creative Commons Attribution 4.0 International License. Read Full License 


\section{Abstract}

The need for wastewater treatment is progressively rising as the release of copious amounts of industrial wastewater is increasing. Likewise, there is an urgent requirement for renewable energy sources because of the growing energy demand and depletion of fossil fuels. The use of microalgae to convert toxic phenolic wastewater to lipid-enriched biofuel has recently been proposed. Here, we report a new strategy for coupling $\mathrm{N}$-doped $\mathrm{TiO}_{2}$-coated photocatalytic optical fibers and a microalgal biofilm to degrade 4chlorophenol (4-CP) and produce biomass. In the combined photocatalysis and biodegradation system, the photocatalytic products were directly biodegraded by the heterotroph-enriched (Salinarimonas and Pseudomonas) biofilm, promoting biomass production; $\mathrm{O}_{2}$ produced by the phototrophs (Scenedesmus obliquus) promoted the generation of hydroxyl free radicals using $\mathrm{N}$-doped $\mathrm{TiO}_{2}$. Thus, the combined photocatalysis and biodegradation system rapidly and sustainably degraded 4-CP while maintaining the growth of the microalgal biomass. The 4-CP removal, dechlorination, and biofilm growth rates reached $\sim 78 \mu \mathrm{M} / \mathrm{h}, \sim 41 \mu \mathrm{M} / \mathrm{h}$, and $1.8 \mathrm{~g} / \mathrm{h} / \mathrm{m}^{2}$, respectively. Overall, we present a useful synergy between an optical catalyst and a bioreactor that has implications for both wastewater remediation and sustainable microalgal biomass production.

\section{Introduction}

4-Chlorophenol (4-CP) is a typical toxic chlorinated organic pollutant in phenol wastewater that causes serious pollution and damage to the environment (Lan et al. 2017). Long-term consumption of water contaminated by 4-CP can cause neurological diseases, such as dizziness, rashes, and itching (Samet et al. 2010 ). Therefore, the rapid and continuous removal of 4-CP from wastewater to protect the ecological environment and public health has become an urgent issue worldwide.

Intimately coupled photocatalysis and biodegradation (ICPB) can effectively degrade phenolic pollutants (Yu et al. 2017; Zhou et al. 2018; Yusoff et al. 2020). This technology uses photocatalysts to degrade refractory compounds, and the partial photocatalytic products are consumed as a carbon source by microorganisms (Rittmann. 2018; Li et al. 2020). Furthermore, it overcomes the shortcomings of incomplete photocatalytic degradation and long-term degradation of biofilms, and thus greatly improves the toxic organic wastewater degradation efficiency. Although the reported ICPB technology has many advantages, the photocatalyst carriers (sponges, porous ceramics, and foams) have poor optical properties, severe light attenuation, and restrictions on the transfer of light energy. Furthermore, bacterial biofilms barely produce oxygen, and even consume it (Al-Amshawee et al. 2020; Carré et al. 2020); thus, an effective coupling between photocatalysis and biofilm technologies is difficult to achieve. This limits the degradation and mineralization of toxic organic wastewater using ICPB technology.

Here, we present a novel ICPB reactor using $\mathrm{N}$-doped $\mathrm{TiO}_{2}$ coated hollow quartz optical fibers (HQOFs) and Scenedesmus obliquus (S. obliquus) as the initial biofilm for the biodegradation of 4-CP (Figs. S1S2). The photoreactor comprises both upper and lower regions for biodegradation and photocatalysis, respectively. The biodegradation of 4-CP and its photocatalytic products releases $\mathrm{O}_{2}$ via photosynthesis. 
The produced $\mathrm{O}_{2}$ is transferred to the photocatalytic region to promote photocatalysis for the production of a strong oxidizing group ( $\mathrm{OH}$ ). The produced ${ }^{\circ} \mathrm{OH}$ rapidly detoxifies 4-CP for use by the biofilm. The conditions required to obtain rapid and continuous degradation were determined by investigating the effects of the dissolved oxygen (DO) concentration, temperature, and $\mathrm{pH}$ on the photocatalytic degradation of 4-CP. For comparison, use of the isolated photosynthetic microalgal biofilm for 4-CP degradation was also studied. Ultimately, the coupled photocatalytic and biodegradation system was used to degrade 4-CP. Furthermore, the changes in the bacterial population in the biofilms were analyzed, and the biomass production and oil accumulation in the biofilms were revealed.

\section{Experimental}

\subsection{Fabrication of photocatalyst and photocatalytic optical fiber}

The experimental materials and reagents for the fabrication of the photocatalyst and photocatalytic fibers are shown in Supplementary Section S1. First, $\mathrm{N}$-doped $\mathrm{TiO}_{2}$ (NT) was prepared according to the method reported by Asahi et al. (2001). The prepared NT powder $(2.375 \mathrm{~g})$ was added to a $0.167 \mathrm{mM}$ acetylacetone solution and ground for $2 \mathrm{~h}$. The ground sample was transferred to $40 \mathrm{~mL}$ of absolute ethanol to prepare a mixed solution. Next, $2.5 \mathrm{~mL}$ of Triton X-100 and $0.3 \mathrm{~g}$ of polyethylene glycol were added to the mixed solution. The mixed solution was stirred continuously for $12 \mathrm{~h}$ and referred to as the NT photocatalytic sol.

The dipping method was used to coat the NT photocatalytic sol onto the cleaned HQOFs. The procedure for cleaning the HQOFs can be found in Zhong et al. (2019). The sol was coated on the cleaned HQOFs at ambient temperature $\left(\sim 25^{\circ} \mathrm{C}\right)$; subsequently, the coated HQOFs were dried in a vacuum drying oven (DZF-6210, JSM, Nanjing, China) at $60{ }^{\circ} \mathrm{C}$ for $15 \mathrm{~min}$. This coating process was repeated 3-5 times. Finally, the HQOFs with NT were dried at $200^{\circ} \mathrm{C}$ for $12 \mathrm{~h}$ to obtain the $20-\mu \mathrm{m}$-thick NT-coated photocatalytic hollow quartz optical fibers (PcHQOFs, see Fig. S1).

\subsection{Preparation and operation of the ICPB photoreactor}

The ICPB photoreactor (length, width, and height of 140, 59, and $27 \mathrm{~mm}$, respectively) was fabricated using polymethyl methacrylate, as shown in Supplementary Fig. S2. It is separated into the upper region (biodegradation region) and lower region (photocatalytic region) using a nuclear pore membrane. The working volumes of the upper and lower regions were $\sim 27$ and $57 \mathrm{~mL}$, respectively. The nuclear pore membrane was used to immobilize the $S$. obliquus cells and transmit the visible light emitted by the photocatalytic optical fiber surface, thus facilitating the production of $\mathrm{O}_{2}$ by $\mathrm{S}$. obliquus photosynthesis, which promotes the generation of photocatalytic products via the degradation of 4-CP in the photocatalytic region. The upper region was composed of a microalgal biofilm and gas phase space, and was used to degrade the photocatalytic products and part of the 4-CP to produce $\mathrm{O}_{2}$. The lower region was composed of two rows of 36 photocatalytic optical fibers, filled with 4-CP wastewater, and used to 
rapidly degrade and dechlorinate the 4-CP. The excitation light within the photocatalytic optical fibers was obtained from UV-vis LED light sources.

In the isolated photocatalysis test, the circulation of the $100-\mathrm{mL} 4-\mathrm{CP}(388.9 \mu \mathrm{M})$ solution in the photocatalytic region of the ICPB photoreactor was controlled at a flow rate of $1 \mathrm{~mL} / \mathrm{min}$ via the wastewater inlet and outlet. The initial pH of the 4-CP solution was adjusted to $5.0-10.0$, using $\mathrm{HNO}_{3}$ and $\mathrm{NaOH}$ solutions, as required. The solution temperature was adjusted to $25-50{ }^{\circ} \mathrm{C}$ using a thermostatic water bath (DCW-0530, Shunmatech, China). High-purity oxygen (99.995\%) was supplied to the ICPB photoreactor via an external oxygen supplier. The oxygen supply per unit time was controlled within the range of 0 to $8.39 \mathrm{mM}$ using a mass flow meter (Rheonik RHM 007, Germany). The 36 photocatalytic optical fibers were then evaluated for their ability to photocatalytically transform 4-CP when illuminated using a UV-vis LED light source with an average irradiance of $20 \mathrm{~W}$ at $360-380 \mathrm{~nm}$ and $30 \mathrm{~W}$ at $400-750$ $\mathrm{nm}$.

For the isolated biodegradation test, the ICPB system with biofilm was cultured, as shown in Supplementary Section S1, and was employed to evaluate the degradation of 4-CP. The 36 optical fibers without photocatalysis were illuminated with visible light (average irradiance of $50 \mathrm{~W}$ at $400-750 \mathrm{~nm}$ ). The circulation of the 100-mL 4-CP solution (initial concentration of $388.76 \mu \mathrm{M}$ ) in the photocatalytic region of the ICPB photoreactor was also controlled at a flow rate of $1 \mathrm{~mL} / \mathrm{min}$ through the wastewater inlet and outlet. The temperature and initial $\mathrm{pH}$ of the 4-CP solution were adjusted in the range $25-45^{\circ} \mathrm{C}$ and $5.0-10.0$, respectively.

In the ICPB system test, the ICPB system comprising 36 photocatalytic optical fibers and biofilm was used to evaluate the degradation of 4-CP. The setup was the same as that used in the isolated photocatalysis test, except that the biofilm was inoculated on the nuclear pore membrane, and the initial $\mathrm{pH}, \mathrm{DO}$ concentration, and temperature were controlled at $7.0,388.9 \mu \mathrm{M}$, and $30^{\circ} \mathrm{C}$, respectively.

\subsection{Characterization techniques}

The HQOF and PCHQOF characterization, bioinformatics analysis, and liquid-phase analysis are detailed in Supplementary Sections S3-S5.

\section{Results And Discussion}

Figure 1a-b show the surface morphology of uncoated HQOF and NT-coated PcHQOF, respectively. Figure $1 b-c$ reveal that the dense and uniform NT photocatalyst was successfully coated on PcHQOF. Figure $1 \mathrm{~d}$ shows the porous structure of the NT coating. Figure $1 \mathrm{e}$ shows that the NT photocatalyst particles had a uniform size with an average of $\sim 30 \mathrm{~nm}$.

Supplementary Fig. S3a and Fig. $1 \mathrm{f}$ characterize the elemental composition of the NT using TEM-EDS plane scans and XRD and XPS analyses. Ti, $\mathrm{O}$, and $\mathrm{N}$ are clearly observed in the NT STEM-EDS plane scans. The XRD analysis of the NT sample showed nine main peaks corresponding to solid diamonds 
(indicated by solid diamonds in Supplementary Fig. S3a) at $2 \theta=25.26,38.01,48.09,53.91,55.07,62.7$, $68.8,70.23$, and 75.09, which correspond to the characteristic diffraction peaks of anatase $\mathrm{TiO}_{2}$. Figure $1 \mathrm{f}$ shows two strong peaks at $458.25 \mathrm{eV}$, and $529.5 \mathrm{eV}$, corresponding to the characteristic peaks of Ti2p, and 01s, respectively. The N 1s XPS spectrum of NT exhibits a characteristic peak at $399.1 \mathrm{eV}$, revealing the $\mathrm{N}$ linkage in $\mathrm{TiO}_{2}$ (interstitial $\mathrm{N}$ ). Together, the results shown in Fig. 1 and Supplementary Fig. S3 demonstrate that NT has been successfully synthesized.

Supplementary Fig. S4a shows the UV-vis absorption spectrum of the NT particles, which reveals a broad absorption edge in the visible region; with the band edge redshifted to $500 \mathrm{~nm}$ owing to defect states created by N-doping, enabling sub-bandgap absorption. Supplementary Fig. S4b shows that the surface luminous spectrum of the NT-coated PcHQOFs was lower than that of the HQOFs in the spectral range 200-750 nm, due to absorption by the NT coating. By contrast, the NT-coated HQOFs exhibited high visible luminous intensity in the spectral range of 750-950 nm, which was caused by the increase in the refractive index of the NT coating. The good light absorption by the NT coating and the surface luminosity of NT-coated PcHQOFs can enhance the photocatalytic activity of the PcHQOFs (Wu et al. 2020) and provide light energy for photosynthesis in the biofilm.

\subsection{Effects of temperature, initial $\mathrm{pH}$, and $\mathrm{DO}$ content on photocatalysis}

Figure S5a and S5b show that the NT-coated HQOFs exhibited the highest photocatalytic degradation of 4-CP at a temperature of $35^{\circ} \mathrm{C}$ and an initial $\mathrm{pH}$ of 7.0. Fig. S5c shows that part of the 4-CP was degraded, as evidenced by the $\sim 67 \%$ loss of $4-\mathrm{CP}, \sim 42 \%$ loss of the initial dissolved organic carbon (DOC), and $56 \%$ dechlorination, after $10 \mathrm{~h}$. Over the 10-h experiment, the dissolved oxygen (DO) concentration decreased due to the consumption of oxygen for the photocatalytic oxidation of 4-CP (Fig. S5d). The pH also decreased because the 4-CP degradation produced small organic acids. Figure 2a shows that the highest photocatalytic degradation of 4 -CP was obtained at $8.93 \mathrm{mM} \mathrm{DO}$ in $4 \mathrm{~h}$. Figure $2 \mathrm{~b}$ shows $100 \%$ removal of $4-C P, \sim 63 \%$ loss of DOC, and $~ 71 \%$ dechlorination after $14 \mathrm{~h}$. The DOC losses were proportionally less than the loss of 4-CP, indicating that organic residuals remained, which is a desirable feature for the coupling of photocatalysis and biodegradation, providing the residuals are biodegradable.

\subsection{Effects of temperature and initial pH on biofilm degradation of 4-CP}

Figure $\mathrm{S} 6$ shows that the highest biodegradation of 4-CP occurred at a $\mathrm{pH}$ of 7.0 and a temperature of $30^{\circ} \mathrm{C}$, which correspond to the most suitable conditions for S. obliquus growth (Breuer et al. 2013). Figure 3a shows that the degree of 4-CP degradation, DOC removal, and dechlorination corresponded to $\sim 83 \%, \sim 68 \%$, and $\sim 85 \%$, respectively, after $10 \mathrm{~h}$. Figure $3 \mathrm{~b}$ shows that the DO concentration rapidly increased from $234.68 \mu \mathrm{M}$ to $265.31 \mu \mathrm{M}$ over $10 \mathrm{~h}$ due to the photosynthetic production of $\mathrm{O}_{2}$ by $S$. obliquus; however, the $\mathrm{pH}$ was only slightly decreased due to the the biofilm metabolism produced 
organic acids. The production of $\mathrm{O}_{2}$ and stabilization of the $\mathrm{pH}$ are beneficial for photocatalysis because they can enhance the NT surface oxygen vacancy production of ${ }^{\circ} \mathrm{OH}$ (Wang et al. 2021).

\subsection{ICPB system for degradation of 4-CP}

Figure 4a shows the rapid removal of 4-CP, which was accompanied by the release of $\mathrm{Cl}^{-}$corresponding to $84 \%$ of the $\mathrm{Cl}$ in 4-CP. Approximately $99 \%$ DOC removal was achieved after $8 \mathrm{~h}$, whereas all 4-CP had been completely removed after $5 \mathrm{~h}$. Overall, the 4-CP removal, dechlorination, and DOC removal rates reached $\sim 78, \sim 41$, and $\sim 27 \mu \mathrm{M} / \mathrm{h}$, respectively, which were much higher than those obtained with isolated photocatalysis and biodegradation. Figure $4 \mathrm{~b}$ shows that whereas the $\mathrm{pH}$ continuously declined, the DO initially declined due to photocatalysis consumption, but then increased with photosynthetic activity. The key role of the $S$. obliquus metabolism is underscored by the increase in biomass over time (Fig. 4c); the growth rate of biofilm in the presence of 4 -CP reached $1.8 \mathrm{~g} / \mathrm{h} / \mathrm{m}^{2}$. Furthermore, Fig. S7 shows that the PcHQOFs demonstrated a repeatable transformation of $4-\mathrm{CP}$ and generation of $\mathrm{O}_{2}$. The photocatalytic activity of the PCHQOFs was maintained at the same level over eight cycles, as the $\mathrm{N}$ doped $\mathrm{TiO}_{2}$ photocatalyst did not detach due to the use of the Triton X-100 cross-linking agent and polyethylene glycol coated on the chemically cleaned HQOFs (Wu et al. 2020). In addition, the photosynthetic production of $\mathrm{O}_{2}$ and biodegradation of photocatalysis products both increase ${ }^{\circ} \mathrm{OH}$ generation by the PcHQOFs, thus maintaining high photocatalytic activity. Notably, S. obliquus grown in the presence of 4-CP had a normal internal cell structure (Fig. S8a) and the biofilm became enriched in Salinarimonas and Pseudomonas (average relative abundances were $~ 17 \%$ and $\sim 18 \%$, respectively; Fig. S8b). Salinarimonas and Pseudomonas are well known for their metabolism of a variety of biorecalcitrant pollutants (Mcleod et al. 2006). In addition, these heterotrophic microorganisms metabolize 4$\mathrm{CP}$ and consume $\mathrm{O}_{2}$ produced by $\mathrm{S}$. obliquus photosynthesis and produce $\mathrm{CO}_{2}$ for $\mathrm{S}$. obliquus growth. This synergy provides an efficient means to consume photocatalytic products (heterotrophs) and $\mathrm{O}_{2}$ produced (phototrophs) by the biofilm, promoting the generation of more ${ }^{\circ} \mathrm{OH}$. The increase in ${ }^{\circ} \mathrm{OH}$ can quickly degrade the high concentration of phenol to achieve a level within the microalgal biofilm adaptation range, thereby avoiding the long-term toxicity of high concentrations of phenol to algal cells, and contributing to the continued growth of the biofilm (Fig. 4c). The synergistic properties of photocatalysis, biodegradation, and photosynthesis enhance the degradation and mineralization of 4-CP and the growth of biofilm biomass.

\section{Conclusion}

The ICPB system, comprising NT-coated PcHQOFs, an NpM, and a biofilm that includes S. obliquus and heterotrophic microorganisms, provided rapid and continuous removal of 4-CP. S. obliquus in the biofilm provided $\mathrm{O}_{2}$ for photocatalysis and for the biodegradation of 4-CP and its photocatalytic products by Salinarimonas and Pseudomonas. Furthermore, the biodegradation of 4-CP and photocatalytic products enhanced the production of ${ }^{\circ} \mathrm{OH}$ and improved its efficiency, which enhanced photocatalysis. The results 
demonstrated that $100 \mathrm{~mL}$ of $4-\mathrm{CP}$ with an initial concentration of $388.9 \mu \mathrm{M}$ was degraded in $5 \mathrm{~h}$ using the ICPB system, with the biofilm maintaining a high activity and growth rate. Thus, the ICPB system with the NT-coated PcHQOFs establishes synergism among photocatalysis, microalgae, and bacteria. This synergism provides an efficient means to rapidly and continually degrade and mineralize 4-CP and convert it to microalgae biomass.

\section{Declarations}

\section{Disclosures}

There are no conflicts of interest to declare.

\section{Acknowledgements}

We acknowledge the service of Biomarker Technologies, Beijing, China, for the 16S rDNA sequencing analysis. This work was supported in part by the National Natural Science Foundation of China $(51876018,51806026)$ and the Scientific and Technological Research Program of Chongqing Municipal Education Commission of China (KJQN201801117).

\section{References}

1. Al-Amshawee S, Yunus MYBM, Vo DVN, Tran NH (2020) Biocarriers for biofilm immobilization in wastewater treatments: a review. Environ Chem Lett 18: 1925-1945. https://doi.org/10.1007/s10311-020-01049-y

2. Asahi R, Morikawa T, Ohwaki T, Aoki K, Taga Y (2001) Visible-Light Photocatalysis in Nitrogen-Doped Titanium Oxides. Science 293(5528): 269-271. https://://doi.org/10.1126/science.1061051

3. Breuer G, Lamers PP, Martens DE, Draaisma RB, Wijffels RH (2013) Effect of light intensity, pH, and temperature on triacylglycerol (TAG) accumulation induced by nitrogen starvation in Scenedesmus obliquus. Bioresource Technol. 143: 1-9. http://dx.doi.org/10.1016/j.biortech.2013.05.105

4. Carré C, Zanibellato A, Jeannin M, Sabot R, Gunkel-Grillon P, Serres A (2020) Electrochemical calcareous deposition in seawater. A review. Environ Chem Lett 18, 1193-1208. https://doi.org/10.1007/s10311-020-01002-z

5. Lan S, Feng J, Xiong Y, Tian S, Liu S, Kong L (2017) Performance and mechanism of piezo-catalytic degradation of 4-chlorophenol: finding of effective piezo-dechlorination. Environ. Sci. Technol. 51(11): 6560-6569. https://doi.org/10.1021/acs.est.6b06426

6. Li F, Lan X, Wang L, Kong X, Xu P, Tai Y, Liu G, Shi J (2020) An efficient photocatalyst coating strategy for intimately coupled photocatalysis and biodegradation (ICPB): Powder spraying method. Chem. Eng. J. 383:123092. https://doi.org/10.1016/j.cej.2019.123092

7. Mcleod MP, Warren RL, Hsiao WWL (2006) The complete genome of Rhodococcus sp. RHA1 provides insights into a catabolic powerhouse. P. Natl. Acad. Sci. USA. 103(42): 15582-15587. 
https://doi.org/10.1073/pnas.0607048103

8. Rittmann BE (2018) Biofilms, active substrata, and me. Water Res. 132:135-145. https://doi.org/10.1016/j.watres.2017.12.043

9. Samet $Y$, Agengui L, Abdelhédi R (2010) Electrochemical degradation of chlorpyrifos pesticide in aqueous solutions by anodic oxidation at boron-doped diamond electrodes. Chem. Eng. J. 161 (1-2): 167-172. https://doi.org/10.1016/j.cej.2010.04.060

10. Wang T, Zhu Y, Luo Z, Li Y, Niu J, Wang C (2021) Oxygen vacancy confining effect on photocatalytic efficiency of Pt1-black $\mathrm{TiO}_{2}$ single-atom photocatalysts for hydrogen generation and phenol decomposition. Environ Chem Lett https://doi.org/10.1007/s10311-020-01144-0

11. Wu Y, Zhong L, Yuan J, Xiang W, Xin X, Liu H, Luo H, Li L, Chen M, Zhong D, Zhang X, Zhong N, Chang $H(2020)$ Photocatalytic optical fibers for degradation of organic pollutants in wastewater: a review. Environ Chem Lett 1-12. https://doi.org/10.1007/s10311-020-01141-3

12. Yu M, Wang J, Tang L, Feng C, Liu H, Zhang H, Peng B, Chen Z, Xie Q (2020) Intimate coupling of photocatalysis and biodegradation for wastewater treatment: Mechanisms, recent advances and environmental applications. Water Res. 175: 115673. https://doi.org/10.1016/j.watres.2020.115673

13. Yusoff N, Ong SA, Ho LN, Rashid NA, Wong YS, Saad FNM, Khalik W, Lee SL (2018) Development of simultaneous photo-biodegradation in the photocatalytic hybrid sequencing batch reactor (PHSBR) for mineralization of phenol. Biochem. Eng. J. 138: 131-140. https://doi.org/10.1016/j.bej.2018.07.015

14. Zhong N, Chen M, Luo Y, Wang Z, Xin X, Rittmann BE (2019) A novel photocatalytic optical hollowfiber with high photocatalytic activity for enhancement of 4-chlorophenol degradation. Chem. Eng. J. 355: 731-739. https://doi.org/10.1016/j.cej.2018.08.167

15. Zhou D, Dong S, Shi J, Cui X, Ki D, Torres Cl, Rittmann BE (2017) Intimate coupling of an N-doped $\mathrm{TiO}_{2}$ photocatalyst and anode respiring bacteria for enhancing 4-chlorophenol degradation and current generation. Chem. Eng. J. 317: 882-889. https://doi.org/10.1016/j.cej.2017.02.128

\section{Figures}



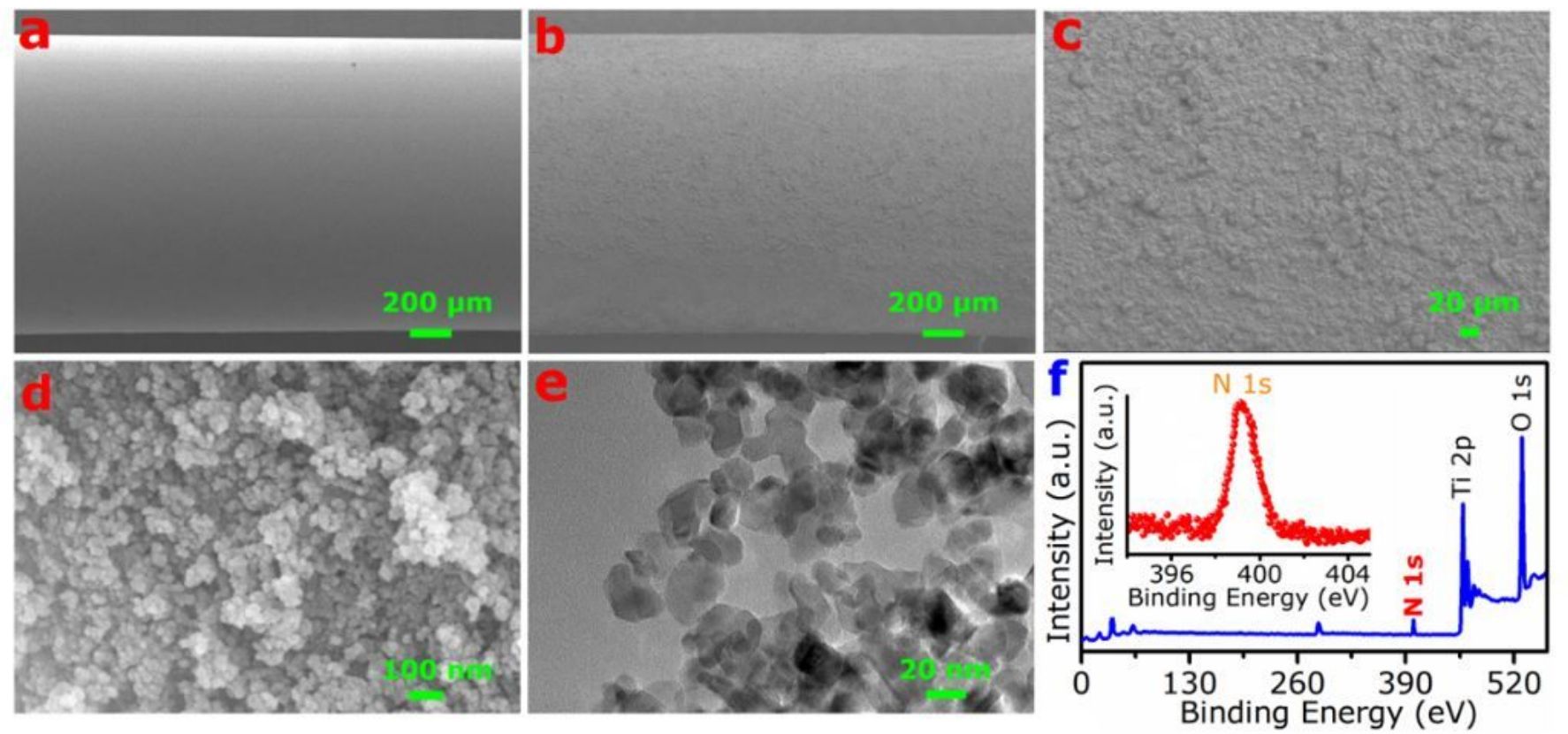

\section{Figure 1}

FESEM, STEM, and XPS images of samples. (a) FESEM image of the HQOF. (b) FESEM image of the NTcoated PcHQOF. (c-d) NT coating under different magnifications. (e) STEM image of the NT particles. (f) XPS spectrum of NT; the inset gives the N 1s XPS spectrum
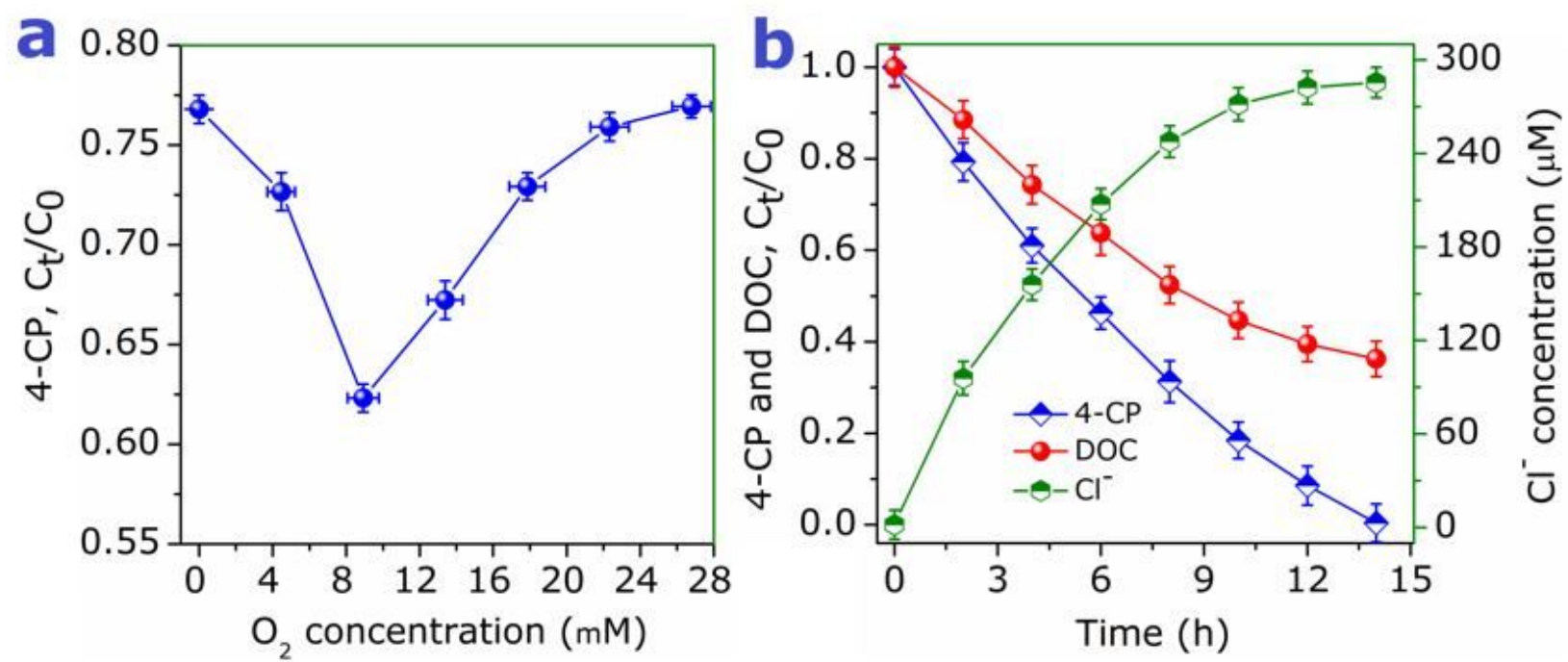

\section{Figure 2}

Photocatalytic degradation of 4-CP. (a) Effect of DO concentration on photocatalytic degradation of 4-CP after $4 \mathrm{~h}\left(\mathrm{pH}=7.0, \mathrm{~T}=35^{\circ} \mathrm{C}\right)$. (b) Losses of $4-\mathrm{CP}$ and $\mathrm{DOC}$ and dichlorination with time $(\mathrm{pH}=7.0, \mathrm{~T}=35$ ${ }^{\circ} \mathrm{C}$, and $\left.8.93 \mathrm{mM} \mathrm{DO}\right)$ 

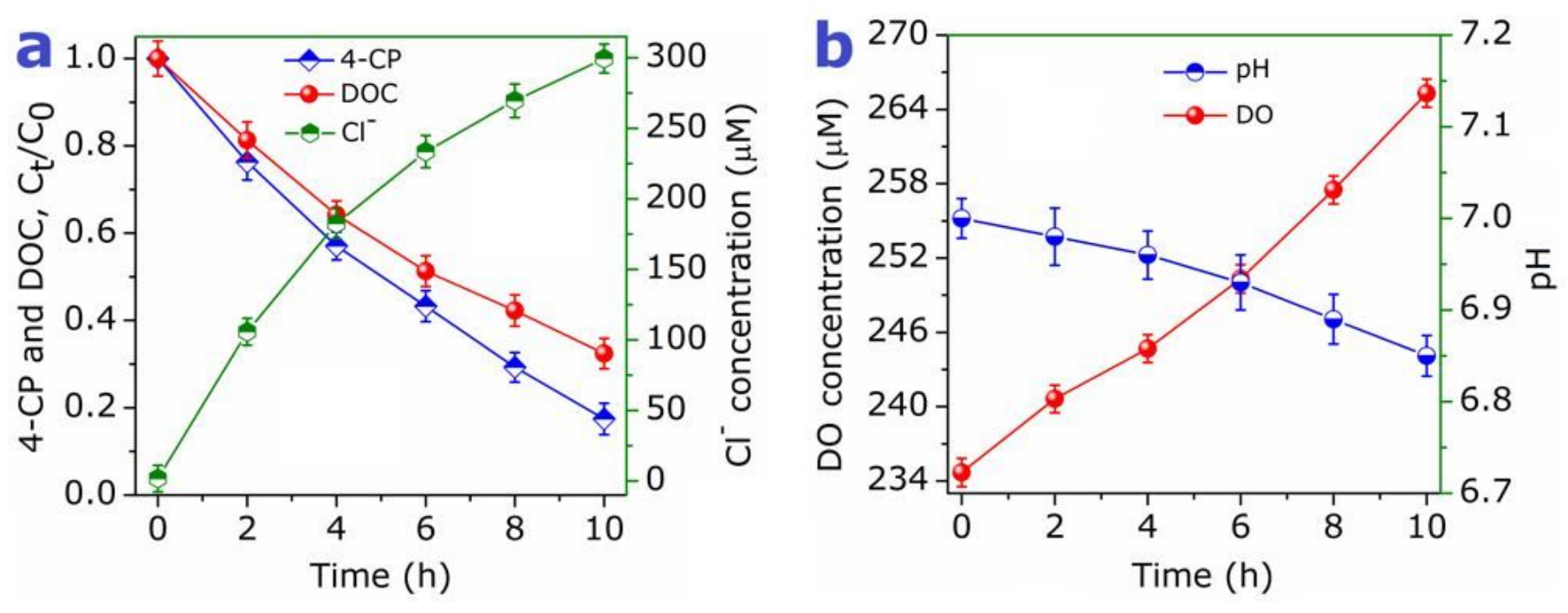

Figure 3

Biofilm degradation of 4-CP and $\mathrm{pH}$ and $\mathrm{DO}$ changes $\left(\mathrm{pH}=7.0, \mathrm{~T}=30^{\circ} \mathrm{C}\right)$. (a) $4-\mathrm{CP}$ and $\mathrm{DOC}$ removal, and dechlorination behavior over time. (b) $\mathrm{DO}$ concentration and $\mathrm{pH}$ changes during the 4-CP removal
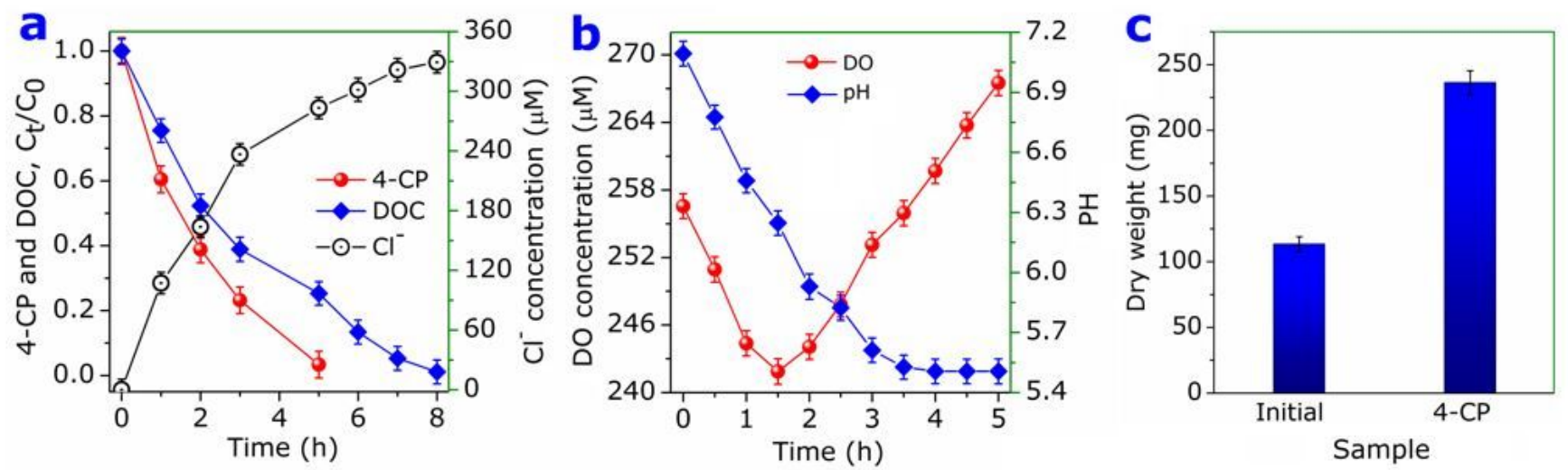

\section{Figure 4}

(a) 4-CP degradation, DOC removal, and dechlorination due to photocatalytic biological close coupling degradation. (b) Change of dissolved oxygen during the coupled degradation of $4-\mathrm{CP}\left(\mathrm{T}=30^{\circ} \mathrm{C}, \mathrm{pH}=7.0\right.$, and $258.44 \mu \mathrm{M}$ initial DO). (c) Dry weight and cell lipid content of the initial biofilm and the biofilm grown with 4-CP for $8 \mathrm{~d}$, respectively

\section{Supplementary Files}

This is a list of supplementary files associated with this preprint. Click to download.

- Supplementaryinformation.docx 